\title{
Gemeinschaft von Verschiedenheiten - religionsphilosophische Vorüberlegungen
}

\section{DORIN ZOSIM OANCEA*}

Communion of diversities. Interconfessional marriages are understood as communions of diversities. It is the author's opinion that the first three chapters of the Book of Genesis offer a model of man's constitutive and simultaneous communion with God and the world. Each person experiences bondings with different theocosmic surroundings or spaces, able to give her/him a special identity. Postparadisiac life is marked by an also simultaneous illegitimate diminishing and enhancing of communion in all directions, included in everybody's bonding system. The communion of diversities, mixed marriages included, participate in this process, which can be overcome only through life in Christ.

Keywords: constitutive communion, image of God dynamics, theocosmic space, diminished or enhanced communion, distorted diversity, bonding

\section{Vorbemerkungen}

Obwohl er im Zusammenhang zwischenchristlicher Mischehen entstand, nimmt sich dieser Beitrag nicht vor, dogmatisch, kirchenrechtlich oder liturgisch zu dieser Frage Stellung zu nehmen, sondern bietet dazu nur Vorüberlegungen eines orthoxen Religionsphilosophen über die Gemeinschaft von zwei verschiedenen Personen, die kommunikatorisch geprägte, von unterschiedlichen Bindungen gekennzeichnete Identitäten aufweisen. Unseres Erachtens stellt dieses das Wesen der Ehe überhaupt dar.

Wir gehen von der Voraussetzung aus, dass Gemeinschaft und Verschiedenheit zwei komplementäre Seinsweisen speziell des Menschen und ganz allgemein der geschöpflichen Welt sind. Christliches Weltverständnis geht aber davon aus, dass diese Struktur nicht innerweltlich, sondern im trinitarischen Dasein des Einen Gottes fundiert ist. Die einzelnen Personen der Hl. Dreifaltigkeit leben in vollständiger Gemeinschaft mit- und untereinander, ohne aber ihre Verschiedenheit zu verlieren, die Kommunikation überhaupt möglich macht. Gott verleiht allen seinen Geschöpfen diese Struktur und deswegen kann man sie auch überall in der uns zugänglichen Welt erkennen. Das ist auch der Grund, warum die hier angesprochene Voraussetzung auch von jenen angenommen werden kann, die den Glauben an Gott nicht haben.

*Pfr. Prof. Dr. i.R. Dorin Zosim Oancea, Universität "Lucian Blaga" in Sibiu, Rumänien; Adresse: 550024 Sibiu - Rumänien, B-dul Victoriei 9, ap.12, E-Mail: oancead@yahoo.com. 
Unsere Ausführungen erfolgen innerhalb eines christlichen Glaubensmodells und wenden sich dem kommunikatorischen Charakter persönlichen Lebens und seiner Einbindung im Gemeinschaftsgefüge zu. Sie möchten dabei nur die Grundstruktur des kommunikatorischen Modells hervorheben, mit dessen Hilfe wir das Verhältnis von Gemeinschaft und Verschiedenheit im Allgemeinen untersuchen. Die Entfaltung des Modells erfolgt eher thetisch, d.h., ausführliche Begründungen der unterschiedlichen Aussagen werden vermieden, einerseits weil der zur Verfügung stehende Raum das nicht erlaubt, andererseits, weil sie zum Gemeingut orthodoxer Glaubenserfahrung gehören, aus ihm entnommen werden. Hinzu kommt die Tatsache, dass, dem religionsphilosophischen Ansatz des Verfassers entsprechend, diese Erfahrung mit neuzeitlichen Erkenntnissen besser vertieft werden kann. Um dieses im Kontext von Gemeinschaft und Verschiedenheit zu veranschaulichen, werden in Kurzfassung die Anhaltspunkte des christlich-orthodoxen Verständnismodells aufgezählt.

Schon auf dieser Ebene kann man zwischen den Sollwerten dieser Gemeinschaft, wie sie unterschiedlichen Texten der Heiligen Schrift zu entnehmen sind, und ihrem Istwert, ${ }^{1}$ also ihrem realen Ausdruck, der unser tägliches Dasein wie auch das unserer Vorfahren kennzeichnet, unterscheiden. Ohne analytisch darauf einzugehen, stellen wir von Anfang an fest, dass die weiter unten angeführten strukturellen und relationellen Komponenten des Modells konstitutiv sind, unabhängig davon, ob es um Soll- oder Istwerte gemeinschaftlicher Existenz geht. Dieser konstitutive Charakter entspricht den Offenbarungstexten, auf die wir zurückgreifen: es sind durchwegs Berichte über Ausgangssituationen, die einen Rahmen weltlicher Existenz und menschlichen Lebens setzen. Ganz allgemein beziehen wir uns dementsprechend auf die zwei Schöpfungsberichte aus Genesis 1-3. Für das Verständnis des Übergangs von den Sollwerten zu den Istwerten werden wir auf das erste Kapitel des Römerbriefes zurückgreifen, was für diese Vorüberlegungen ausreicht. Im Anschluss an die orthodoxe Glaubenserfahrung vertreten wir die Überzeugung, dass das Leben jedes Menschen mit diesen Ausgangspunkten im Dialog steht, z. T. von ihnen bestimmt wird, unabhängig von seiner Positionierung im Ablauf der Geschichte. Aufgrund dieser Synchronisierung

\footnotetext{
1 Handlungstheoretische und systemtheoretische Ansätze unterscheiden zwischen einem angestrebten Sollwert und einem erreichten Istwert. Siehe z. B., neben vielen anderen, Herbert Fuchs, Systemtheorie und Organisation, Wiesbaden, Springer Fachmedien Wiesbaden $\mathrm{GmbH}$ 2013, S. 35; Anette Hiemisch, Eine handlungstheoretische Beschreibung Sozialer Phobien, Pabst, Wolfgang Science 2000, S. 45; Werner Herkner, Psychologie, Berlin, Springer-Verlag 1986, S. 317; Michael Brodowski, Kollektives Lernen als Grundlage organisationalen Lernens, Berlin-Münster-Wien-Zürich-London, LIT 2006.
} 
bewahren die in den Anfängen festgelegten Bedingungen einen konstitutiven Charakter. $^{2}$

\section{Personale Existenz als Gemeinschaft und Verschiedenheit}

\subsection{Sollwerte der Gemeinschaft und Verschiedenheit}

Wie schon früher angedeutet, geht es um ursprünglich gegebene Werte, mit deren Hilfe die vollständige Gemeinschaft Gottes mit den Menschen und damit mit der gesamten Schöpfung verwirklicht werden sollte. Auch nach dem sogenannten Sündenfall sind sie nach wie vor aktuell, wenn man das Heil in Christus als erneute Möglichkeit versteht, die Gemeinschaft mit Gott in Anbetracht dieser Sollwerte zu erfahren. Im Folgenden beziehen wir uns eher schematisch auf die Hauptkomponenten dieses Modells.

Die doppelte Ausrichtung des Menschen. Ausgangspunkt unserer Überlegungen ist die Feststellung, dass, den Schöpfungsberichten aus Genesis 1-3 entsprechend, der Mensch, wie alle anderen Geschöpfe, eine doppelte, konstitutive Ausrichtung aufweist, auf Gott und auf einen anderen Menschen bzw. auf andere Geschöpfe hin. Der Begriff „Ausrichtung“ schließt einerseits einen Kommunikationspartner, andererseits den eigenen Bedarf, mit diesem ein Kommunikationsverhältnis aufzubauen, sowie die Bereitschaft, die Errichtung eines solchen Verhältnisses vorzuschlagen oder anzunehmen, ein.

Die von uns im Zusammenhang der Ausrichtung verwendete Qualifizierung „konstitutiv“, reißt die Kommunikationsbereitschaft aus dem Bereich der freien und oftmals willkürlichen Entscheidung heraus und versetzt sie in die Strenge des Notwendigen. Ganz einfach gesagt, schließt sie die Existenz des Menschen als solche ohne Kommunikation in beide Ausrichtungen aus.

Das in beiden Fällen verwendete Merkmal „konstitutiv“ deutet nicht auf eine inhaltliche, sondern auf eine strukturelle Gleichheit der zwei Ausrichtungen hin, aber nur in dem Sinne, dass sie beide für die Existenz des Menschen unentbehrlich sind. Andererseits weisen sie auf strukturelle und inhaltliche Unterschiede hin, die den jeweiligen Kommunikationspartnern entsprechen, also Gott und den unterschiedlichen Geschöpfen.

Der Mensch ist für die Gemeinschaft mit Gott sowohl strukturell als auch relationell anderswie ausgestattet als für jene mit einem Menschen,

2 Für ein besseres Verständnis dieser Beziehung verweisen wir auf die Synchronisierung liturgischen Lebens mit den hier angesprochenen Anfängen. Am letzten Sonntag vor der Ostern-Fastenzeit, dem Vergebungs-Sonntag, wird in der orthodoxen Kirche die Vertreibung Adams aus dem Paradies nicht nur als etwas Vergangenes, sondern, wegen ihrer Folgen, zugleich Aktuelles vergegenwärtigt. 
selbst wenn diese „Ausstattungen“ bis zu einem gewissen Punkt auch gemeinsame Züge aufweisen. Die Gemeinschaft des Menschen mit Gott setzt den Schöpfer als erstes und den Menschen als zweites Subjekt voraus: Gott fragt, setzt Regeln fest, erwartet vom Menschen, dass dieser Rollenunterschied auch eingesehen wird; der Mensch beantwortet die Anfrage, erkennt die ihm und der ganzen Welt auferlegten Regeln bzw. den Rollenunterschied an. Innerhalb dieses Kommunikationssystems stellt dann auch der Mensch als zweites Subjekt seine eigenen Fragen an Gott und erhält die entsprechenden Antworten. Die Fähigkeit, diesen Rollenunterschied der Subjekte zu erkennen, stellt auch ein Existential, eine transzendentale Veranlagung des Menschen dar, dem sich komplementär ein zweites anschließt, nämlich die Möglichkeit, Gottes Wort, Empfinden und Handeln zu verstehen und darauf zu antworten. Diese zwei Existentialen sind nur innerhalb dieses Kommunikationssystems des Menschen mit Gott legitim, sie verleihen dieser Ausrichtung einen einzigartigen Charakter, der nur hier der schöpfungsmäßig gegebenen Beschaffung und Intentionalität des Menschen, seiner ihm von Anfang an verliehenen Zielorientierung entspricht.

Die vorhin angesprochene Andersartigkeit der Gemeinschaftsmöglichkeiten kommt deutlicher zum Ausdruck, wenn man den zweiten Menschen als Teil bzw. Vertreter der gesamten Welt betrachtet, mit allen darin eingesetzten Geschöpfen, die sowohl quantitativ als auch qualitativ unterschiedliche Komplexitätswerte aufweisen. Dieses führt dann zu unterschiedlichen Gemeinschaftsbeziehungen des Menschen mit der Welt und so entstehen Kommunikationssysteme, denen sich der Mensch, als ihr Subjekt, anpasst, und die jedem Geschöpf helfen, der ihm schöpfungsmäßig verliehenen Soll-Qualität zu entsprechen.

Was die Komplexität des Menschen anbelangt, so kommt hier alles in Betracht, was ihn als leibliches und geistig-geistliches Geschöpf ausmacht. Einerseits alles, was man heute rein wissenschaftlich mit der Leiblichkeit mitdenkt, und viel mehr dazu, andererseits Selbstbewusstsein, Bewusstsein und Unbewusstsein, die ganze Fülle von Affekten und anderen Eigenschaften, die möglicherweise noch unbekannt sind.

Direkte und indirekte Gemeinschaft mit Gott. In den Schöpfungsberichten findet ein Dialog zwischen Gott und dem Menschen statt, woraus man schließen kann, dass zwischen ihnen eine unmittelbare, direkte Gemeinschaft besteht. In der analogen Sprachweise der Offenbarungstexte könnte man sagen, dass es eine Gemeinschaft von Angesicht zu Angesicht ist, ${ }^{3}$ so

3 Seiner Natur entsprechend, befindet sich Gott jenseits einer jeden Erkenntnis und deswegen auch jeder Begrifflichkeit, in der Sprache der Kirchenväter hat er einen absolut apo- 
wie man sie z. B. im Gebet erfährt. Sie wird vom Hl. Johannes Chrysostomus mit Gottessprechen in unserem Bewusstsein zusammengebracht, als Antwort auf unsere Anfragen oder auf moralische Herausforderungen des Alltags, für die wir keine Lösung finden. Am Dialog mit Gott nimmt der ganze Mensch zusammen mit seinen innerweltlichen Beziehungen teil, unabhängig ob er sie anspricht oder nicht.

Darüber hinaus geht es hier um ein an und für sich schwer beschreibbares Ergriffensein, das dem Menschen im eigentlichen Sinne nur innerhalb dieser Kommunikation mit Gott zugänglich ist. Analog kann man sie jedoch mit bestimmten Zügen zwischenmenschlicher Beziehungen vergleichen, selbst wenn sie bereits entformt und verfremdet sind, wie man es weiter unten sehen wird. Aufgrund dieser Erfahrungen und der Art und Weise, wie diese Gemeinschaft in den Offenbarungstexten erscheint, hat Rudolf Otto ein misterium fascinans und ein misterium tremendum als legitime Grundeinstellungen des Menschen im Rahmen seiner direkten Gemeinschaft mit Gott identifiziert.

Es gibt aber auch eine indirekte Gemeinschaft des Menschen mit Gott, einerseits als Erfüllung Seiner Gebote, andererseits während der innerweltlichen Kommunikation, solange die ununterbrochene Gemeinschaftt mit Gott nicht nur für den Menschen, sondern für alle Geschöpfe konstituiv ist.

Exemplarisch für den Ablauf der Kommunikation über ein Gebot, ist die gesamte Situation um die verbotene Frucht: in einem ersen Schritt gebietet Gott dem Menschen, etwas innerhalb seiner Beziehungen mit einem anderen Geschöpf zu tun; der Mensch gehorcht oder gehorcht nicht; Gott stellt das eine oder das andere fest und damit ist dieser indirekte Kommunikationszyklus abgeschlossen. Nach dieser Verifizierung und anhand ihrer Ergebnisse, initiiert Gott einen nächsten Kommunikationszyklus, usw.

Laut Genesis 1,28 empfängt der Mensch von Gott einen dauernden Auftrag, der den Gesamtsinn seines Lebens ausmacht: er soll über die ganze

phatischen Charakter. Die Offenbarung als solche, dereren Subjekt er ist, erweist seinen personalen oder kataphatischen Charakter, dem Merkmale eigen sind, die bis zu einem gewissen Punkt jenen der kreatürlichen Welt ähneln. Von diesen Gemeinsamkeiten ausgehend, verwendet man auch in Bezug auf Gott eine Begrifflichkeit, die aus dem innerweltlichen Sprachgebrauch des Menschen entnommen ist. Dabei sollte man niemals vergessen, dass es um eine analoge Sprechweise geht, deren Inhalt Gottes Eigenschaften nicht genau bezeichnet, sondern sich ihnen nur annähert. Siehe in dieser Hinsicht die Erläuterungen des Johannes Chrysostomos zum Gehen Gottes, Prinz Max, Herzog zu Sachsen (Hrg.), Des hl. Johannes Chrysostomus Homilien über die Genesis oder das erste Buch Mosis, Paderborn, Druck und Verlag von Ferdinand Schöningh 1913, S. 192. 
Welt herrschen, was in der von uns verwendeten Begrifflichkeit nichts anderes bedeutet, als mit allen Geschöpfen Kommunikationszyklen einzuleiten, die alle gleichzeitig ebenso viele Strukturen indirekter Gemeinschaft mit Gott darstellen.

Die indirekte Gemeinschaft kommt auch dadurch zustande, dass der Mensch in jedem Geschöpf dessen Gemeinschaft mit Gott auf allen Ebenen seiner eigenen Persönlichkeit wahrnimmt. In unserem reellen Leben erschließt sich dieses zunächst nur verstandesmäßig auf, aber bei vertiefter Gemeinschaft mit Christus wird es allmählich zu einer Erfahrung der gesamten Person, innerhalb eines Vorgangs, für den die Orthodoxen den Namen theosis verwenden.

Die Gemeinschaft des Menschen mit der Welt. Der schöpfungsmäßig gegebenen Ausrichtung des Menschen auf die Welt hin entsprechen Relationen mit ihr, die eine bestimmte Struktur aufweisen, je nach der Eigenart des betreffenden Geschöpfes. Wir sagten schon, dass diese Relationen konstitutiv, also lebensbestimmend sind. Der Mensch benötigt sie, ganz einfach um als Mensch leben zu können. Wenn sie ausfallen, hört sein konkretes Dasein auf. Dieses ist leicht verständlich, wenn man die Beziehung zur Welt z. B. mit Hilfe der Atmung oder Ernährung veranschaulicht. Bleibt Luft oder Nahrung aus, setzt Atem- oder Nahrungsnot ein, was dann die Notwendigkeit des Atmens oder des Sich-Ernährens impulsartig ans Licht bringt. Diese physiologischen Notsituationen sind für die gesamte Gemeinschaft des Menschen mit der Welt beispielhaft, selbst wenn in manchen Bereichen der Drang zur Erfüllung dieser Kommunikationsmöglichkeit und die Konsequenzen seiner Nichterfüllung weniger deutlich sind als in diesen speziellen Fällen.

Aus dem hier Gesagten kann man schließen, dass zu einer konstitutiven Ausrichtung auch eine Dynamik gehört, unter Umständen mit imperativem, impulsartigem Charakter. Zu dieser dynamischen Einstellung wird der Mensch in Genesis 1,28 aufgerufen, als Gott ihm den Herrschaftsauftrag erteilt. Diese Dynamik ist allen Kommunikationsausrichtungen eigen und bedeutet, angesichts der indirekten Gemeinschaft des Menschen mit Gott, aktive Verantwortung für die höchstmögliche Gemeinschaft aller Geschöpfe mit Ihm. In diesem Sinne kann John Zizioulas behaupten, dass der Mensch ein "Priester der Schöpfung" sein muss. ${ }^{4}$ Gleichfalls Priester zwischenmenschlicher Beziehungen, die in ihrer Solldimension dementsprechend immer auf gegenseitige Verantwortung Gott gegenüber ausgerichtet sind.

4 John Zizioulas of Pergamon, Proprietors or Priests of Creation, http://www.orth-transfiguration.org/proprietors-or-priests-of-creation/, abgerufen am 15. September 2018. 
Verschiedenheiten. Genesis 2,21-24; 3,20 berichten in der historisch gebundenen, aber metaphorischen Sprache, die die Schöpfungsberichte durchwegs bis zu den Anfängen Abrahams (Gen, 11, 10 ff) gebrauchen, von einer Modulation des Menschlichen, in dem Sinne, dass ein zweiter Mensch eingeführt wird. Die zwei sind sich zwar sehr ähnlich, von hier auch die Gleichheit des Gattungsnamens, aber gleichzeitig auch voneinander verschieden, was von den unterschiedlichen Personennamen angezeigt wird. Eine ähnliche Verschiedenheit wird in Genesis 1-3 von anderen Lebewesen nicht dargestellt, aber im Zusammenhang der Sintfluterzählung erscheinen die Tiere paarweise $($ Gen.7,2), so dass man davon ausgehen kann, dass Verschiedenheit alle Geschöpfe kennzeichnet. ${ }^{5}$

Es gibt aber nicht nur eine Extension, sondern auch ein Intension der Verschiedenheit, d. h., jede einzelne Existenzebene eines Menschen und alle miteinander, in ihrer Aufeinanderbezogenheit, sind davon durchdrungen. In diesem Gefüge wird die Verschiedenheit von jedem einzelnen Element, z. B. vom affektiven Leben, getragen, und alle zusammen finden sich in seiner Einzigartigkeit. Diese kommt auch im Zuge der direkten und indirekten Gemeinschaft mit Gott und der Welt zum Ausdruck. Diese werden vom Mann anderswie erfahren als von der Frau, was auf keinen Qualitätsunterschied, sondern eben auf grundsätzliche, legitime Verschiedenheit hinweist.

Ebenbildlichkeitdynamik. Der Genesis 1,26 entsprechenden Aussage wurde der Mensch nach Gottes Bild, ihm ähnlich geschaffen. In der orthodoxen Tradition werden die zwei Begriffe nicht als bedeutungsgleich, sondern als Grundelemente in einem Werde-Prozess betrachtet, den wir als Aktualisierung des Potentiellen verstehen. Auch dieses Werden gehört zur konstitutiven Gemeinschaft des Menschen mit Gott, so dass die Anfrage des Herrschaftsauftrags in der Ebenbilddynamik beantwortet wird, die ihrerseits durch das ununterbrochene Eingreifen Gottes durch direkte und indirekte Gemeinschaft zustande kommt. Alle bereits angesprochenen Kommunikationsmöglichkeiten sind dabei ständig aufeinander bezogen, so dass der Mensch einerseits von sich aus ständig höhere Gemeinschaftsebenen anstrebt, diese andererseits durch Gottes Handeln ermöglicht werden. In diesem Prozess wird die Verschiedenheit des Einzelnen gleichzeitig gestärkt und er ist in der Lage, die Verschiedenheit des Dialogpartners in einem neuen Licht zu erkennen, was dann wiederum neue Horizonte der Gemeinschaft nach allen Richtungen hin eröffnet. Man könnte sagen, dass die Verschiedenheit im Zuge der Ebenbildlichkeitsdynamik zusammen mit der vereinheitlichenden

5 Voaussetzung der geschöpflichen Verschiedenheit ist jene der Hl. Dreifaltigkeit, die im Schöpfungsakt als Struktur, einer jeden Existenzebene entsprechend, weitergegeben wird. 
Gemeinschaft in jeder Hinsicht weiter vertieft wird, so dass sie von einer Stufe zur anderen immer deutlicher ausgeprägt sind. Fortgeschrittene Ebenbildlichkeitsdynamik bedeutet keinesfalls Einebnung der Verschiedenheit zugunsten der Einheit, sondern betonte Einheit und Verschiedenheit in ihrer Komplementarität.

Die etappenweise gestaltete Ebenbildlichkeitsdynamik schließt dieses kommunikatorische Gefüge ein und verleiht somit auch der Verantwortung des einen Menschen für den anderen schrittweise neue qualitative und quantitative Werte. Dieses bedeutet, dass die Sollwerte der Kommunikation sich auch dementsprechend ändern, so dass der Mensch in dieser Hinsicht ständig zwischen dem Vergangenen und dem Jetzigen schwebt und ständig auf das Zukünftige dieser Sollwerte hin orientiert ist, die ihm von Gott auf jeder Stufe neu offenbart werden.

Theokosmischer Raum. Das ist ein für unsere Argumentation wichtiger Begriff, der die bis jetzt eingeführten Komponenten des Modells zusammenfügt. Er ist einerseits für menschliche Gemeinschaft kennzeichnend, schließt aber auch jene mit Gott und der Welt ein, beide im Horizont der Ebenbildlichkeitsdynamik. Hervorzuheben ist auf jeden Fall zunächst die Tatsache, dass ein theokosmischer Raum die Beziehung zwischen wenigstens zwei Menschen voraussetzt, die ihrerseits am gesamten Kommunikationsgefüge auf allen Ebenen teilnehmen. Mit der Einführung des Paares Mann-Frau wird etwas Grundsätzliches ausgesagt: Voraussetzung einer jeden Kommunikationsform überhaupt ist die Verschiedenheit der Teilnehmer, so dass die Schöpfungsberichte das Entstehen eines ersten theokosmischen Raumes mit Hilfe dieser zwei sich unterscheidenden Menschen begründet, erst ihre Verschiedenheit macht zwischenmenschliche Gemeinschaft möglich.

Aus den Schöpfungsreferaten ist es ersichtlich, dass die schon angesprochene doppelte Orientierung des Menschen (eigentlich aller Geschöpfe) auf Gott und auf die Welt hin, sowie die doppelte Gemeinschaft des Menschen mit Gott eigentlich schon eine zweifache Nuancierung des ursprünglichen theokosmischen Raumes bedingt. Einerseits sehen wir die Menschen in einem Dialog mit Gott, während dem auch das innerweltliche Leben des Menschen nicht nur angesprochen, sondern auch als indirekte Gemeinschaft mit dem Schöpfer erfahren wird. Andererseits ist es deutlich, dass der Mensch mitten in der Welt lebt, als deren Gestalter, aber dieses ohne sich von Gott zu trennen, sondern, ganz im Gegenteil, als Priester der Schöpfung, der ihre vertiefte Gemeinschaft mit dem Schöpfer fördert. Eigentlich ist es in diesem Fall derselbe theokosmische Raum, mit zwei unterschiedlichen Schwerpunkten und entsprechenden Ausdrucksformen, die letztlich zwei komplementäre Unter-Räume bilden. 
Der erste theokosmische Raum, in dem die konstitutiven Merkmale menschlicher Existenz in ihrer Verbundenheit offenbart werden, weist noch etwas Bemerkenswertes auf, was vielleicht nachparadiesische Entwicklung vorwegnimmt, aber gerade für diese paradigmatische Bedeutung bekommt. Damit meinen wir die Bildung neuer theokosmischer Räume aus bereits bestehenden. In Gen. 2,24 werden die drei Elemente einer Grundstruktur aufgezählt: „Darum wird ein Mann seinen Vater und seine Mutter verlassen und seiner Frau anhangen, und sie werden sein ein Fleisch." Ein erster Raum erscheint in aller Deutlichkeit: Vater, Mutter und Mann. Im zweiten Fall werden die Eltern der Frau nicht erwähnt, sondern eher vorausgesetzt. Der dritte theokosmische Raum entsteht offensichtlich durch die Verbindung der zwei ersten, deren Verschiedenheit sowohl mit Hilfe der unterschiedlichen Namen, als auch der ganzen Dramatik ausgedrückt wird, die dem Zeitwort „verlassen“ eigen ist. ${ }^{6}$ Es sollte nicht vergessen werden, dass in diesen drei Fällen der Schwerpunkt zwar auf Innerweltlichkeit liegt, sie aber in Bezug auf Gott auch einen komplementären Ausdruck der direkten Gemeinschaft mit Gott kennen, selbst wenn der damit verbundene Dialog der zwei Menschen mit Gott schon ein negatives Vorzeichen hat.

Neben dieser Annäherung von zwei theokosmischen Räumen gibt es noch die von der Ebenbildlichkeitsdynamik bedingte Entwicklung eines jeden einzelnen bzw. der drei hier Erwähnten. Ihr zufolge führt jeder neue Schritt in der Verwirklichung des Herrschaftsauftrags zu einem ganz neuen Zustand des einen oder anderen theokosmischen Raumes, so dass ein jeder von ihnen einerseits mit sich selbst identisch, andererseits hingegen ganz neu ist. Das führt selbstverständlich zur Möglichkeit unterschiedlicher Entwicklungen, wie es auch das Entstehen des dritten Raumes andeutet, das die zwei vorgehenden nicht einfach summiert, sondern, bei allen Gemeinsamkeiten, eine neue Wirklichkeit darstellt. Das paradiesische Paradigma schließt ihre Widersprüchlichkeit aus, diese wird aber zum Kennzeichen nachparadiesischer Realitäten. Auf Grund der Entstehungsmöglichkeit neuer theokosmischer Räume durch Übergang von einem zum anderen und der ununterbrochen einsetzenden Ebenbildlichkeitsdynamik innerhalb eines jeden Raumes, ist die Bedeutung eines letzten Grundzugs der kommunikatorischen Existenzweise, den wir noch besprechen möchten, ersichtlich.

6 Gerade diese Episode hebt den hermeneutischen Schlüsselcharakter der Schöpfungsberichte hervor. Dem patriarchalischen Charakter altisraelischer Gesellschaft entsprechend, hätte die Frau und nicht der Mann das Elternhaus verlassen müssen. Siehe dazu Gerhard von Rad, Das erste Buch Mose: Genesis, Göttingen,Vandenhoeck \& Ruprecht 1987, S. 60. Unseres Erachtens beschreibt Gen. 2,24 keinen zur Zeit der Textverfassung geltenden sozialen Tatbestand, sondern das Wesen zwischenmenschlicher Beziehungen im allgemeinen Gemeinschaftskontext, den wir untersucht haben. 
Bindungen. Alle Geschöpfe trachten danach, ihren kommunikatorischen $\mathrm{Zu}-$ stand, von dem sie übrigens abhängig sind, zu bewahren, sie sind homeostatisch orientiert. Das gilt auch für den Menschen, der gleichfalls an seinen Beziehungen hängt und sich an seine eigene Identität bis in die Tiefen seiner Person gebunden fühlt. Diese tiefe Bindung beeinflusst emotionales und vernunftmäßiges Urteilen, also alle Entscheidungen, die der Einzelne bezüglich seiner gemeinschaftlichen Einbeziehung trifft. Ihre Bedeutung erscheint in den Schöpfungsberichten eher in verkappter Form, im Zusammenhang des Sündenfalls und aller anderen Ereignisse, die der paradigmatischen Geschichte der Menschen Gestalt verleihen. Diesem kommunikatorischen Modell entsprechend, ist die Bindung des Menschen nicht statisch bzw. endgültig, sondern entspricht den unterschiedlichen Schritten innerhalb seiner Ebenbildlichkeitsdynamik. Das heißt also, dass jeder Fortschritt in der Verwirklichung des Herrschaftsauftrages eine neue, im jeweiligen Kontext individuell geprägte Bindung verursacht, was gleichzeitig deutlich macht, dass jedem dieser Schritte eine neue ontologische Ebene entspricht. Es ist offensichtlich, dass beim Übergang von einer Etappe der Ebenbildlichkeitsdynamik zur anderen die jeder Bindung eigene Trägheit zum Teil überwunden wird, was dazu führt, dass die neu entstandene Bindung die alten bis zu einem gewissen Punkt einschließt und ihnen gegenüber gleichzeitig als ein selbstständiges Novum erscheint.

Wir haben im Kontext theokosmischer Räume die Möglichkeit des Übergangs von einem bestehenden zu einem neuen Raum kurz angesprochen. Man kann die Frage stellen, ob die Gültigkeit der Bindungen somit nicht infrage gestellt wird. Unsere Antwort geht davon aus, dass die Bindungen aus dem ursprünglichen Raum auch in dem neu entstandenen weiter wirken, wenn auch mit verminderter Kraft. Im Schöpfungsbericht wird nicht behauptat, dass der Mann und die Frau auf die Gemeinschaft mit ihren Familien verzichten, sondern dass sie eine eigene Gemeinschaftsstruktur aufbauen, die vom Weiblichen entscheidend geprägt wird. Auf dieser Ebene der Sollwerte besteht zwischen den unterschiedlichen Bindungsstrukturen der Gemeinschaftsdynamik theokosmischer Räume kein Widerspruch, sondern ein Ineinander, das mit konzentrischen Kreisen vergleichbar ist: der äußerste umfasst die inneren, weist also eine höhere Komplexität auf, die aber mit den vorausgehenden Formen nicht in Widerspruch steht.

\subsection{Istwerte der Gemeinschaft}

Die Schöpfungsberichte erzählen eine Geschichte der Menschen und der Schöpfung, in der Soll- und Istwerte bis zu einem gewissen Punkt übereinstimmen, was nur dann möglich und verständlich ist, wenn Grundmerkmale des Soll-Seins auch im Ist-Sein fortbestehen, zu einem gewissen Grad 
wenigstens. Dieselben Berichte eröffnen aber auch eine andere Ereignisfolge, die bie heute andauert und in der diese Übereinstimmung wenigstens zum Teil fehlt, obwohl sie für das Sein des Menschen unentbehrlich ist. Der Bruch ist unter dem Namen Sündenfall bekannt und hat als Inhalt eine gewaltige Verformung der vorgegebenen Kommunikationszyklen und der Einstellung des Menschen seinem Schöpfer und der Welt gegenüber.

\section{Das Fortbestehen konstitutiver Elemente der Gemeinschaft. Genesis 3 kennt zwei} Hauptausrichtungen: einerseits den Sündenfall, andererseits die Festlegung neuer Existenzbedingungen für den Menschen. Bemerkenswert ist die Gemeinschaft, die auch nach dem Sündenfall zwischen Gott und dem Menschen besteht. Sie ist zwar brüchig geworden, der Mensch versteckt sich vor Gottes Angesicht, er ist nicht mehr imstande, seine eigenen Fehler einzusehen. Die ursprünglich gegebene, für uns eigentlich unverständliche Unmittelbarkeit direkter Kommunikation von Angesicht zu Angesicht ist verschwunden und auch sind ihm die Geschöpfe, z. B. die Feigenblätter, mit denen die kategorialen Vorfahren ihre neu entdeckte Nacktheit bedecken, und die Bäume des Paradieses, hinter denen sie sich verstecken, für die indirekte Gemeinschaft mit Gott nicht mehr transparent ${ }^{7}$. Dem Vierergespräch aus Genesis 3,9-16 kann man aber entnehmen, dass der Mensch von seinem Schöpfer weiterhin angesprochen wird und fähig ist, ihm zu antworten, selbst wenn seine Worte schon eine neue, von ihm jetzt verzerrte Wirklichkeit gestalten. Auch nach dem Sündenfall ist der Mensch in der Lage, mit Gott in Gemeinschaft zu bleiben, wenn auch in einer verminderter Form. Die Schaffung neuer Existenzbedingungen für den Menschen und das ganze Handeln Gottes nach der Verweisung des Menschen aus dem Paradies zeigen wiederum, dass Gott diese verminderte Gemeinschaft ununterbrochen erneut und sie dem Menschen ermöglicht. Mit der Aussicht ihrer Vervollkommnung durch die Gemeinschaft mit Jesus Christus, seinem menschgewordenen Sohn, wie Genesis 3,15, orthodoxer Glaubenserfahrung entsprechend, zu lesen ist.

Die Ausführungen des nachfolgenden Unterkapitels vorwegnehmend, möchten wir die Tatsache betonen, dass auch unter den schwierigsten Umständen, nach schlimmsten Entscheidungen, jeder Mensch den theokosmischen Charakter eigener Existenz bewahrt, also in ständiger Gemeinschaft mit dem Schöpfer bleibt, selbst dann, wenn er dieses nicht mehr einsieht. Von hier ausgehend, können wir weiterhin feststellen, dass alle im Zusam-

\footnotetext{
7 Gen.3, 7: „Da wurden ihnen beiden die Augen aufgetan und sie wurden gewahr, dass sie nackt waren, und flochten Feigenblätter zusammen und machten sich Schurze"; Gen.3, 8b: „Und Adam versteckte sich mit seiner Frau vor dem Angesicht Gottes des Herrn zwischen den Bäumen im Garten." Alle Zitate aus der Hl.Schrift werden der Lutherbibel 2017, Deutsche Bibelgesellschaft, entnommen.
} 
menhang der Sollwerte erwähnten Strukturen, Relationen und Prozesse, die die unterschiedlichsten Gemeinschaftsformen gestalten, in ähnlicher Weise fortbestehen, eben weil sie zur Satzung des Menschen gehören, weil sie für ihn konstitutiv sind. So ist es zu verstehen, dass sie also da sind, aber in verminderter Gestalt. Die nachparadiesischen Menschen streben weiterhin nach Gemeinschaft mit Gott und der Welt, können nicht isolliert leben; sie erhoffen sich ein besseres Leben und sind bereit, sich mühevoll dafür vorzubereiten; sie gliedern sich in theokosmische Räume ein und sind bestrebt, selber neue zu gründen; sie sind an ihre individuelle und kollektive Identität gebunden und möchten sie auch nicht verlieren, sind sogar bereit, dafür schwerwiegende Opfer zu bringen, letztlich das eigene Leben herzugeben.

Verminderungen und falsche Aufwertungen der Gemeinschaft. Wenn die Kontinuität ursprünglicher Gemeinschaftsformen die helle Seite nachparadiesischen Lebens darstellt, so bilden die Verminderung der Gemeinschaft und die falschen Aufwertungen sicherlich eine Schattenseite, die der Mensch aus eigenen Kräften nicht überwinden kann. Das ist wenigstens die Aussage der Offenbarung Gottes in Jesus Christus und die Überzeugung seiner Anhänger, so wie sie in der Tradition der Kirchenväter fesgelegt wurde ${ }^{8}$. Ein deutliches Verständnis dieses Geschehens findet man in Römer 1,22 - 23,25, ${ }^{9}$ wo der Sündenfall als unrechtmäßige Neuorientierung von Kommunikationsqualitäten verstanden wird: das, was Gott gebührt, wird auf Menschen und andere Geschöpfe übertragen. Aus der Beziehung zu Gott wird Entscheidendes weggenommen und den Geschöpfen zugewendet, so dass man diesen eine Ehrerbietung erweist und eine Qualität zuspricht, die ihnen auf keinen Fall zukommt.

Aus Genesis 3,8 - 19 geht hervor, dass diese Umwendung nicht vollständig war und ist, dass, trotz ihrer Verminderung, die Kommunikation zwischen Gott und dem Menschen weiterhin besteht: sie sprechen miteinander, der Mensch versteht die neuen Lebensregeln, die ihm Gott festsetzt und die seinen ersten nachparadiesischen theokosmischen Raum gestalten.

8 Für Judaismus und Islam sind die Folgen des Sündenfalls bei Weitem nicht so radikal: in manchen Fällen kann der Mensch durch genaue Einhaltung bestimmter, von Gott offenbarter Vorschriften, der vollkommenen Gemeinschaft mit ihm wieder teilhaftig werden, also ein Gerechter sein. Eine ähnliche Argumentation wurde im Christentum, berechtigterweise oder nicht, Pelagius zugeschrieben. Sie wurde $418 \mathrm{im}$ vom Hl. Augustin einberufenen Konzil von Carthago und 431 im Ökumenischen Konzil von Ephesus verurteilt.

9 „Die sich für Weise hielten, sind zu Narren geworden / und haben die Herrlichkeit des unvergänglichen Gottes vertauscht mit einem Bild gleich dem eines vergänglichen Menschen und der Vögel und der vierfüßigen und der kriechenden Tiere. / ... Sie haben Gottes Wahrheit in Lüge verkehrt und das Geschöpf verehrt und ihm gedient statt dem Schöpfer, der gelobt ist in Ewigkeit." 
Dessen ungeachtet halten wir fest, dass es keine vollständige Gemeinschaft mehr gibt, dass ein entscheidender Teil davon auf das Geschöpfliche in jedwelcher Form umgeleitet wurde. Diese zwei Vorgänge möchten wir näher betrachten.

Wir hatten von Anfang an hervorheben können, dass alles, was auf der einen Kommunikationsebene erfolgt, komplementäre Konsequenzen auf der anderen hat. Verminderung der Gemeinschaft mit Gott führt also zur verminderten Gemeinschaft mit den Menschen und den anderen Geschöpfen; Ehrung der Geschöpfe, als ob sie dem Heiligen angehörten, führt zur Ausstattung Gottes mit menschlichen, überhaupt mit geschöpflichen Zügen, wie der Hl. Geist es mit Hilfe des Apostels im Römerbrief überaus deutlich ausdrückt. Die zwischengeschöpflichen Beziehungen werden vom Menschen dementsprechend auch diesem Paradigma untergeordnet, so dass man zweifelsohne behaupten kann, dass die Verminderung aller Gemeinschaftsformen und die falsche Aufwertung bzw. Entwertung aller Teilnehmer am kommunikatorischen Geschehen zwischen Gott und der Welt, die gesamte nachparadiesische Existenz der Menschen kennzeichnen.

In gleicher Weise ist selbstverständlich auch die direkte und indirekte Gemeinschaft des Menschen mit Gott von diesem, für den nachparadiesischen Menschen konstitutiven Geschehen betroffen. Die Akzente können von Fall zu Fall unterschiedlich sein. Bei ausgeprägter Frömmigkeit könnte man meinen, dass sich nichts geändert hätte, was sicherlich nicht stimmt. Die entgegengesetzte Extremsituation verneint jedwelche Beziehung zu Gott, direkt und indirekt, was wiederum täuschend ist, solange laut neutestamentlicher Offenbarung diese Gemeinschaftsformen für alle Geschöpfe, also auch für alle Menschen, konstitutiv sind. Unzählige Schattierungen gibt es zwischen den zwei Extrempositionen, die insgesamt wieder und wieder dieses eine bestätigen: die Istwerte aller Menschen, zu allen nachparadiesischen Zeiten, leiden auch in dieser Hinsicht unter unvermeidlichen Verminderungen und falschen Aufwertungen.

Diese zwei unmittelbar miteinander verbundenen Vorgänge führen zu einem ganz neuen, z. T. falschen Selbstverständnis des Menschen und einer dementsprechend verformten Kommunikationsrealität, die für die nachfolgende Entwicklung des Menschen kennzeichnend werden sollten und von Gottes schöpferischer Absicht weit entfernt sind. Es ist ein grundlegendes Fehlverhalten, das vom Schöpfer nicht durch die Vernichtung des schuldigen Geschöpfes, sondern, ganz unerwartet, durch die Festlegung von ganz neuen Lebensbedingungen für den Menschen beantwortet wird, die von dessen prinzipieller Fehlbarkeit ausgehen. Gleichzeitig wird aber auch die Perspektive der endgültigen Heilung eröffnet, wenn der Kopf der Schlange 
bzw. des Bösen als Urheber einer jeden Gemeinschaftsminderung und falscher Aufwertung zerschmettert werden soll. ${ }^{10}$

Die neuen Lebensbedingungen gestalten einen gleichfalls neuen theokosmischen Raum, der die konstitutiven Merkmale des vorangehenden bewahrt, aber sie gleichzeitig mit unausweichbaren Verminderungen und falschen Aufwertungen aller menschlichen Identitäten und mit den dazu gehörenden Kommunikationsmöglichkeiten versetzt. Diesem nachparadiesischen Anfang entstammen alle anderen theokosmischen Räume, die Geschichte gestalten und von ihr gestaltet werden, und die von den zwei ursprünglichen Schwerpunkten, der Gemeinschaft mit Gott und jener mit anderen Menschen und der Welt, getragen werden. In diesem Sinne erkennen wir vorwiegend auf die Gemeinschaft mit Gott orientierte Räume, in denen jene mit anderen Menschen, zwar im Hintergrund, aber durchaus präsent ist. Sie stellen den Ort individueller und kollektiver Erfahrungen religiös-geistlichen Charakters dar und finden erkennbaren Ausdruck in der Vielfalt der Religionen. Zum zweiten Strang gehören alle theokosmischen Räume, in der eben die Gemeinschaft mit den anderen Menschen und der Welt im Vordergrund und jene mit Gott im Hintergrund steht: Staat, Volk, Stadt, Dorf, Familie, Schule, kollektiver Arbeitsplatz, Freundeskreis usw. Es ist nun wichtig, in Erinnerung zu bringen, dass die zwei Grundausrichtungen des Menschen zu allen Zeiten komplementär sind. Auf die theokosmischen Räume bezogen bedeutet dieses erstens, dass jeder von ihnen, wie alle Menschen, die ihn gestalten, gleichfalls doppelt ausgerichtet ist, und zweitens, dass auch sie komplementär sind, dass sie sich gegenseitig beeinflussen, dass man auf dieser Ebene von einer communicatio idiomatum sprechen kann. Religiöse Erfahrungen eines Menschen beeinflussen sein Leben in der Welt und umgekehrt. Dasselbe gilt auch für die entsprechenden theokosmischen Räume, z. B. für eine Familie, die ihre religiöse Zugehörigkeit aktiv wahrnimmt. Ihre Mitglieder zeichnen sich gerade dadurch auch innerhalb der säkularen Gemeinde aus. Sind die Mitglieder einer Familie hingegen zu sehr auf das Weltliche konzentriert, so wirkt sich dieses auch auf ihr religiöses Verhalten aus.

Theokosmische Räume unter nachparadiesischen Bedingungen. Auch unter ihnen wird die/der Einzelne ununterbrochen von der konstitutiven Gemeinschaft mit Gott und der Welt geprägt, und zwar den Vorbedingungen des theokosmischen Raumes entsprechend, in den sie/er hineingeschaffen wird und in dem ihre/seine Sozialisierung erfolgt. Der Mensch lebt seit aller

10 Gen. 3,15a: „... ich will Feindschaft setzen zwischen dir und der Frau und zwischen deinem Samen und ihrem Samen; er wird dir den Kopf zertreten ....". Der Text wird in der orthodoxen Tradition weiterhin auf das erlösende Werk Christi bezogen und als Protoevangelium betrachtet. 
Ewigkeit in Gottes Gedanken. Aber dieser Gedanke wird in einem ganz bestimmten Augenblick konkret, wenn der Mensch in einen bestimmten theokosmischen Raum hineingeboren wird. ${ }^{11}$ Und seit dem Augenblick an wird er einerseits in dessen spezifische Gemeinschaft mit Gott und andererseits in dessen innerweltliche Dynamik einbezogen. Hineingeboren in ein umfassendes Gebilde, das eine ganz bestimmte Struktur mit dazu gehörenden Relationen zwischen den Komponenten aufweist, und in dem sie/er sozialisiert wird. Die Sozialisierung erfolgt im Einklang mit den Altersstufen und fängt schon im pränatalen Leben des Fötus an. In diesem Stadium menschlichen Lebens werden dem noch im Leibe der Mutter lebenden Kind über die primäre Verbindung mit seiner Mutter und die sekundäre mit der Umwelt, die mindestens in ihrer akustischen Dimension auch deutlich nachweisbar ist, grundlegende Informationen vermittelt, mit denen die Grundstruktur seines gesamten zukünftigen Lebens nach der Geburt gezeichnet wird. ${ }^{12}$ Wir gehen davon aus, dass auch die konstitutiven Gemeinschaftsstrukturen und ihre Verzerrungen in diesem Lebensstadium weitergegeben werden, so dass kein Mensch bei seiner Geburt in dieser Hinsicht eine tabula rasa darstellt.

Der gesamte Vorgang der Sozialisierung steht unter dem Zeichen kommunikatorischer Verminderungen und Aufwertungen, von Anfang an und in beide Ausrichtungen. D. h. zunächst, dass vornehmlich die Mutter ihre religiösen und innerweltlichen Erfahrungen, die schon entscheidend beeinträchtigt sind, dem Fötus vermittelt. Das erfolgt nicht inhaltlich, sondern strukturell, diese schon geformten Informationen zeichnen Strukturen, die nach der Geburt weiter ausgebaut werden. Aus ihrer Verbindung mit den eigenen geistlichen und biologischen Vorgaben, die von Gott und den Eltern kommen, gestalten sie einen Unterbau für die Sozialisierung des Kindes nach seiner Geburt und für die Entwicklung der betreffenden Persönlichkeit. Es ist gleichzeitig das konkrete Fundament der Ebenbildlichkeitsdynamik, das diese von der Mutter, also aus der Welt kommenden Einflüsse, mit

11 Wichtige Überlegungen dazu aus orthodoxer Sicht findet man bei Dumitru Stăniloae, Ortodoxie şi Românism, Sibiu, Tipografia Arhidiecezană 1939.

12 Das pränatale Leben des Menschen wird schon seit fast einem Jahrhundert und in den letzten Jahrzehnten immer deutlicher dokumentiert. Zur Kommunikation mit dem Fötus mit Hilfe akustischer Signale und deren Wirkung nach der Geburt des Kindes siehe z. B. Alessandra Piontelli, Vom Fetus zum Kind: Über den Ursprung des psychischen Lebens. Eine psychoanalytische Beobachtungsstudie, Stuttgart, Klett-Cotta 1996. Aufklärend für die Kontinuität der Strukturen zwischen dem pränatalen und postnatalen Leben ist z. B. Barbara Jakel, „Pränatale Wurzeln der Psychotherapie - eine bindungstheoretische Perspektive“, in: The International Journal of Prenatal and Perinatal Psychology and Medicine, 20 (3,4/2008). Die in Heidelberg beheimatete „International Society of Prenatal and Perinatal Psychology and Medicine“, welche dieser Fragestellung interdisziplinär nachgeht, verbreitet regelmäßig das weltweit erworbene Erfahrungs- und Erkenntniswissen. 
dem Sein Gottes im Gedanken Gottes verbindet und dadurch von Anfang an identitätsprägend wirkt.

Das Kind nimmt auch nach der Geburt die schon erwähnten Einflüsse auf, in den ersten Monaten weiterhin vorwiegend von der Mutter, wobei auch die Rolle der anderen Familienmitglieder, insbesondere des Vaters, zunimmt, die gemeinsam seinen ersten postnatalen theokosmischen Raum bilden. In der ganzen Zeit bis einschießlich dem siebenten Lebensjahr wird die Kommunikationsstruktur auf dem bereits pränatalen Fundament innerhalb dieses Raumes aufgebaut. Es entsteht somit eine Grundstruktur, die für das gesamte Leben der betreffenden Person bestimmend ist und bis zum Lebensende die Grundmerkmale dieses ersten Raumes trägt. Vertreten sind dabei immer die konstitutiven Kommunikationsstrukturen und Relationen, in ihrer schon angesprochenen Doppelorientierung, mit den unumgänglichen Verminderungen legitimer Gemeinschaft und den falschen Aufwertungen, die am Anfang nur aus der Umgebung stammen, danach aber auch vom Kind, dem Jugendlichen oder dem Erwachsenen gleichfalls unumgänglich produziert werden.

Wir haben im paradiesischen Zusammenhang feststellen können, dass jeder theokosmische Raum eine Gemeinschaft von Verschiedenheiten ist. Es wurden dort schon Verschiedenheiten der theokosmischen Räume angesprochen, aus denen ein neuer entstehen kann und die, gemeinschaftlich verbunden, einen übergeordneten Raum bilden. Das Vorzeichen nachparadiesischer Existenz ist aber negativ. Das Aufnehmen von Verminderungen und falschen Aufwertungen, die für den Raum eigener Sozialisierung kennzeichnend sind, und die „Eigenproduktion“ des Kindes, Jugendlichen, Erwachsenen heben die Tatsache hervor, dass die Verschiedenheit der einzelnen Personen bzw. der theokosmischen Räume, in die sie hineingeschaffen werden, die sie aber auch mitgestalten, von Anfang an entstellt ist. Zunächst durch Verminderung der Kommunikation auf unterschiedlichen Ebenen und Bereichen: Verlust an Vertrauen in die eigenen Kräfte, Unmöglichkeit richtiger Kommunikation mit sich selbst, mit anderen Menschen und den Geschöpfen aus dem eigenen und den restlichen theokosmischen Räumen; nicht zu vergessen das allmähliche Aufgeben einer umfassenden Gemeinschaft mit Gott, so dass sie oberfächlich wird und oftmals bewusst nur noch als Ritual überlebt, wenn überhaupt. Hinzu kommen ebenfalls vielfältige falsche Aufwertungen, die das eigene Selbstgefühl, die Ehrfurcht vor einem Mitmenschen oder vor Geschöpfen manchmal ins Unendliche steigern können, so dass man in ihnen nicht mehr das sieht, was sie tatsächlich sind, sondern Vertreter von fast übermenschlichen, ja göttlichen Mächten. 
Bindungen. Am Ende unserer Überlegungen über Sollwerte hatten wir die Bedeutung theokosmischer Bindungen angesprochen, die für den Menschen konstitutiv sind und seine Verschiedenheit festigen, also zu seinem Wesen gehören. Wir möchten diesen Gedanken auch hier aufnehmen und damit unsere Überlegungen im Zusammenhang der Istwerte beenden.

Die wesentliche Rolle von Bindungen für den inneren Aufbau der Menschen, die Tatsache, dass sie der Ebenbildlichkeitsdynamik entsprechen, sowie die Möglichkeit, manche Bindungen aufzugeben und neue einzugehen, wurden bereits hervorgehoben. Vielleicht sollte gerade jetzt ihre dynamische Rolle in der Struktur der theokosmischen Person erwähnt werden. Wir hatten hervorgehoben, dass die Öffnung zur Gemeinschaft und die gleichzeitige Betonung eigener Verschiedenheit für jeden Menschen konstitutiv sind. Dies bedeutet, dass sie/er daran gebunden ist. Beide Haupttendenzen des Menschlichen haben gerade deswegen einen dynamischen Charakter und das stimmt dann selbstverständlich auch für die entsprechenden Bindungen. Es ist also nicht nur von einem eher passiven Zusammenhalten die Rede, sondern von einer Dynamik, die jeder Bindung eigen ist, unabhängig ob sie nach außen oder nach innen orientiert ist. Diese Erkenntnis gilt sicherlich zunächst für den paradiesischen Menschen, ist aber für das Verständnis des nachparadiesischen Menschen von zwingender Notwendigkeit.

Um dieses Verständnis des nachparadiesischen Menschen im Kontext seiner Bindungen zu vertiefen, möchten wir zusätzlich einige Elemente hervorheben, die uns dafür notwendig erscheinen.

Als erstes sei hier die Hierarchie der Bindungen angesprochen, die sowohl für die einzelne Person als auch für den theokosmischen Raum, in dem sie lebt, bedeutsam ist. Ohne diesem Gedanken ausführlich nachzugehen, verweisen wir aus der allgemeinen Erfahrungswelt auf einige Bindungen, die für das Sein des Menschen als solches wesentlich sind. Im Vordergrund steht zunächst die Bindung zwischen Mutter und Kind, unabhängig vom Alter der beiden. In den letzten Jahrzehnten hat man viel darüber gesprochen, insbesondere im Kontext des pränatalen, des perinatalen Lebens des Menschen und seiner ersten Kindheitsjahre.

Andere wichtige Bindungen sind jene zum etwas weiteren theokosmischen Raum, in den man hineingeboren und sozialisiert wurde, also die Familie. Dabei meinen wir damit das tiefgehende und zugleich umgreifende Gefühl von Zugehörigkeit, von Zusammenklang mit der inneren, unverwechselbaren Harmonie, die für den jeweiligen Raum kennzeichnend ist, und die manchmal auch als Disharmonie empfunden wird. Auch hier findet man dann hierarchische Einordnungen theokosmischer Räume: Familie, Gemeinde, ethnische und staatliche Zugehörigkeit, wobei deren Rei- 
henfolge Änderungen erfahren kann. Ähnlich sind auch andere komplexe Gegebenheiten, wie Schule, Wahlverwandschaften jedwelcher Art, die der/ dem Einzelnen ein Spezifikum verleihen, das für sie/ihn lebensbestimmend werden kann. In gleichem Maße kann die religiöse Zugehörigkeit zu einer sozialen Gruppe mit deutlich gezeichneter Identität oder ihr Fehlen für das eigene Selbstverständnis von entscheidender Bedeutung sein. In allen aufgezählten Fällen steht die Zugehörigkeit zu einem bestimmenten theokosmischen Raum im Vordergrund, die Bindung konzentriert sich darauf und die betreffende Person nimmt zu jedem Zeitpunkt dessen Verschiedenheit wahr, die ihn von anderen theokosmischen Strukturen gleicher Art unverwechselbar unterscheidet.

Innerhalb eines bestimmten theokosmischen Raumes und von seinen Eigenarten getragen, erscheinen im Falle aller daran Beteiligten selbstverständlich auch andere Schwerpunkte, die ihre Verschiedenheit ausmachen: die eigenen Vorzüge, Unzulänglichkeiten und Empfindlichkeiten, Neigungen, die man pflegt usw., letztlich das eigene Selbstgefühl. Die Mitglieder einer Familie z. B. nehmen schon ihre bis zu einem Punkt einzigartigen Gemeinsamkeiten wahr, sind sich aber auch ihrer Eigenarten, ihrer Verschiedenheit bewusst und möchten auch als solche wahrgenommen werden.

Bindungen konzentrieren sich immer auf die Verschiedenheiten einer Person und eines theokosmischen Raumes, in den sie gemeinschaftlich eingebunden ist. Oftmals werden diese Verschiedenheiten bewusst als Teil einer Struktur wahrgenommen, die man für sich oder den eigenen theokosmischen Raum als wesentlich betrachtet. Es kann aber auch sein, dass diese Verbindung zum eigenen Ganzen einem nicht bewusst ist, dass die Bindung mehr oder weniger ohne eine treffende Erklärungsmöglichkeit ganz einfach da ist. Die bestehenden Verbindungen bleiben im Unterbewusstsein, was ihre Wirkungskraft keinesfalls mindert.

Die prä- und postnatale Sozialisierung wurde als Fundament der Ebenbildlichkeitsdynamik bezeichnet, was zur falschen Schlussfolgerung führen könnte, dass diese danach gut funktioniert. Die weiteren Ausführungen werden bereits gezeigt haben, dass dies nicht der Fall ist. In diesem Zusammenhang sollte man aber noch einen Aspekt in Betracht ziehen, und zwar, dass diese Dynamik konstitutiv ist. Dies bedeutet, dass die verbliebenen Strukturen authentischer Gemeinschaft nach Weiterentwicklung drängen, dass der Mensch auch im Guten bis zu einem bestimmten Punkt fortschreiten kann. In gleicher Weise drängen aber auch die Verminderungen der Gemeinschaft nach noch weniger, und die falschen Aufwertungen nach mehr und noch mehr! Dieses Weniger oder Mehr wird dann in die Tiefen der eigenen Persönlichkeit aufgenommen und als etwas Selbstverständliches 
betrachtet, sie werden zu einer festen Bindung, die nur mit viel Mühe aufgegeben werden kann.

Im Falle paradiesischer Bindungen konnte man eine gewisse Beweglichkeit feststellen, die das homöostatische Verweilen auf den nacheinander folgenden Stufen in der Ebenbildlichkeitsdynamik nicht endgültig sein lässt, sondern das legitime Entstehen neuer theokosmischer Räume ermöglicht. Nachparadiesische Entwicklungen führen die konstitutive Anhänglichkeit bis zu einem gewissen Punkt in legitimer Form weiter, verleihen aber auch diesen die unübersehbare Prägung von nichtlegitimen Entwertungen und Aufwertungen. Andere wiederum werden in großem Maße entstellt, mit ungeahnten Konsequenzen für das Selbstverständnis der betreffenden Person und für ihre daraus folgenden Handlungsweisen. Aus diesem Grund kann man in manchen Fällen Bindungen an legitime Identitätsmerkmale finden, andere werden wiederum neu ausgerichtet, zugunsten überbewerteter Schwerpunkte, was die Homöostatik des Ganzen und der individuellen Segmente durcheinanderbringt. Dramatische Erfahrungen, wie ein Erdbeben, eine Überschwemmung oder ein Krieg, eine schwere Erkrankung, ein Familiendrama, die eigene Auswanderung oder jene der Angehörigen usw. können oftmals solche Neupositionierung verursachen, die schwerwiegende Folgen haben können, sowohl für eine Einzelperson, als auch für einen kleineren oder größeren theokosmischen Raum. Ähnliche Ereignisse können aber auch das Gegenteil erreichen, in dem Sinne, dass jemand mit stark entwerteten oder aufgewerteten Bindungen in der Lage ist, nochmals bewusst oder unbewusst neue, legitime Verbundenheit aufzubauen.

Unabhängig davon, ob sich eine Person ihrer Identitätsverbundenheiten bewusst ist oder nicht, weisen diese eine innere Struktur auf, die dann für ihr Selbstverständnis, für ihren Sitz im Leben, für die Art und Weise, wie sie sich dem Drang zur Gemeinschaft und jenem zur Verschiedenheit gegenüber verhält, verantwortlich ist. Man kann also von einer Hierarchie der Bindungen sprechen, die sowohl das Verhalten der einzelnen Personen als auch theokosmischer Räume entscheidend beeinflussen. Wenn diese legitime Hierarchie beschädigt ist, wenn z. B. die Bindungen zum Dienst an Gott in der Familie oder in der Schöpfung, zu allgemeinen und speziellen Werten und Rechten des Menschen verloren geht, dann wird sie mit anderen ersetzt, die eine andere, schiefe Wertekonstellation als Ziel haben. Dann hört der Mensch auf, ein Priester der Schöpfung zu sein und versteht den Herrschaftsauftrag nur noch als willkürliche Nutznießung; dann gibt er möglicherweise den Glauben an die Relevanz seiner Gemeinschaft mit Gott für sein Leben in der Welt zum Teil oder sogar vollständig auf und wendet sich ungehindert einer vollkommenen Diesseitigkeit zu, usw. In manchen Fäl- 
len führt dies zu einer unbewussten Neugestaltung von alten Erinnerungen, wodurch die neue Konstellation der inneren Person und deren Handlungen nach außen gerechtfertigt werden. Solche Neugestaltungen können dann die individuelle oder/und kollektive Person zum Fundament jedwelcher Art, chauvinistischen Nationalismen, radikalen Globalismen usw. werden lassen.

\section{Verschiedenheiten in Gemeinschaft}

Die Gemeinschaft der Verschiedenheiten haben wir schon wiederholt angesprochen, sodass unsere nachfolgenden Überlegungen Manches vom Gesagten wiederholen. Gleich von Anfang an soll daran erinnert werden, dass in den theokosmischen Räumen die Gnade Gottes ununterbrochen mitwirkt und den Lauf der Dinge mitbestimmt. Wir sagten früher, dass alle Menschen, wie alle anderen Geschöpfe, seit immer schon in Gottes Gedanken leben und zur für sie vorgesehenen Zeit weltliche Gestalt bekommen. Diese Verbindung zwischen dem Leben des Menschen im Gedanken Gottes und in der Welt bezieht sich, orthodoxer Schöpfungstheologie entsprechend, nicht nur auf den Eintritt in dieses irdische Sein, sondern auch auf dessen gesamten Ablauf. Also auch auf die Bildung des Bindungskomplexes jedes einzelnen Menschen, auf Verschiedenheiten und die Art und Weise, wie sie konkret in Gemeinschaft treten. Dieser ganze Vorgang ist gleichzeitig ein Teil der direkten und indirekten Gemeinschaft des Menschen mit Gott. Andererseits erteilt Gott dem Menschen den Herrschaftsauftrag, den dieser im Verlauf einer innerweltlichen Dynamik erfüllt. Diese scheint dem Beobachter vollkommen selbstständig zu sein, obwohl sie mit der Ausrichtung menschlicher Existenz auf Gott hin komplementär ist und mit ihr im ständigen Dialog steht. Wir konzentrieren uns in diesem Kapitel auf die innerweltliche Dynamik, ohne aber die komplementäre Gott-Mensch aus dem Auge zu verlieren.

Weiterhin möchten wir darauf aufmerksam machen, dass die nachfolgenden Überlegungen sich ausschließlich mit der Struktur des Vorgangs und nicht mit möglichen Wahrheitsansprüchen der an ihm beteiligten Personen oder theokosmischer Räume befassen. Einige der weiter unten angeführten Beispiele zum Verhältnis zwischen kleineren und größeren theokosmischen Räumen könnten den Eindruck erwecken, dass in ihrer Beziehung zur Minderheit die Mehrheit moralisch, wirtschaftlich und politisch immer im Unrecht stünde. Das ist nicht gemeint, weil es beobachtungsmäßig nicht stimmt und unserer eigenen Sichtweise nicht entspricht. Eine ausreichende Zahl von Beispielen zeigt, dass das Unrecht oftmals von der Minderheit ausgeht und die Mehrheit die Leidende ist.

Dieselbe Bemerkung gilt auch im Zusammenhang der Wahrheitsfrage. Diese wird überhaupt nicht angeschnitten, weil der hier zur Verfügung 
stehende Raum es auch nicht erlaubt. Es sei dazu nur gesagt, dass die innerhalb eines theokosmischen Raumes herrschende Meinung nicht obsolet ist, wenn in einem anderen eine ganze andere, vielleicht bedeutend neuere dominierend ist. Der Anspruch auf Wahrheit gilt in beiden Fällen und beruht gerade auf derselben Dynamik der Ebenbildlichkeit und der Bindungen, die wir untersuchen. Der Mechanismus ist derselbe, aber die Bindungen nicht, sowohl auf der Ebene der Einzelperson als auch eines ganzen theokosmischen Raumes. Und von daher unterschiedliche Wahrheitsansprüche.

Verschiedenheiten außerhalb einer Familie. Wir haben uns schon auf die Verschiedenheiten jener Personen, durch deren Gemeinschaft der theokosmische Raum der Familie entsteht und besteht, bezogen. Das hat für das Verständnis individueller Verschiedenheit innerhalb eines primären theokosmischen Raumes ausgereicht, darauf werden wir nicht mehr eingehen, obwohl das Thema an sich einladend ist. Unsere Aufmerksamkeit richtet sich auf Verschiedenheiten aus unterschiedlichen theokosmischen Räumen, auf die Faktoren, die ihre Gemeinschaft begünstigen oder verhindern können.

Für den Anfang rufen wir die Tatsache in Erinnerung, dass komplementär zu der nach innen und nach außen ausgerichteten Dynamik der Verschiedenheit eine Dynamik der Gemeinschaft existiert, die in gleichem Maße das Verhalten der Person reguliert, solange die zwei im Gleichgewicht stehen. Unter nachparadiesischen Bedingungen kann eine vollständige Ausgeglichenheit niemals erreicht werden, aber unter allen Umständen sind beide Größen präsent. Auch übermäßig aufgewertete Verschiedenheiten können den Drang nach Gemeinschaft niemals vollständig hemmen und umgekehrt. Was kann nun den Impuls nach Gemeinschaft der Verschiedenheiten fördern und was kann ihn hemmen?

Warum wehren sich Verschiedenheiten manchmal vor neuen Gemeinschaften? Eine zusammenfassende Antwort wäre: wegen der Trägheit homöostatischer Strukturen. Die Verschiedenheit einer Person ist, wie bereits ausgeführt, keine einfache Eigenschaft, sondern die Realität eines Bindungssystems, das als solches funktioniert und nicht geändert werden möchte. Alle Systeme dieser Art sind bis zu einem gewissen Punkt träge, anderswie könnten sie ihre Identität nicht bewahren, sie wären zu versatil dafür. Diese Trägheit ist prinzipiell von Person zu Person verschieden, je nach Veranlagung und Sozialisierung, was dann auch die Rolle der Homöostatik des Raumes hervorhebt. Außerdem unterscheiden sich die Grade der Trägheit von der Stellung der in Frage kommenden Bindung innerhalb des Bindungssystems bzw. der Bindungshierarchie, dem Prinzip entsprechend, dass eine höhere Stellung die größere Trägheit bedingt. (Konkret heißt das z. B., dass man zwischen 
altbewährten und neuen Freundschaften, die jene ersetzen möchten, sich für die ersteren entscheidet, weil sie von einer festen Bindung gesichert sind.)

Von dieser Feststellung ausgehend, finden wir weitere Abwehrreaktionen in unseren nachfolgenden Ausführungen zur Gemeinschaft.

Der Impuls nach Gemeinschaft, die Bereitschaft dazu sind schöpfungsmäßig gegeben und gehören daher zum Wesen des Menschen. Auf dieser Grundlage werden in die für jeden Menschen eigene Hierarchie der Bindungen auf allen Ebenen der Person Gemeinschaft fördernde Faktoren aufgenommen. Fördernd wirkt die Ähnlichkeit oder sogar Übereinstimmung des Bindungssystems aus einem theokosmischen Raum mit jenem eines anderen Raumes. In diesem Fall sind die Verschiedenheiten geringfügig, so dass die Gemeinschaft leicht erreichbar ist. Manche umfassenden theokosmische Räume wie Nachbarschaften, Schulen, Berufsgemeinschaften oder Vereine unterschiedlichster Art, um nur einige davon aufzuzählen, pflegen systematisch Gemeinsamkeiten und die entsprechenden Bindungen, so dass sie besonders einladend für mögliche Gemeinschaften sind.

Auch im Falle von einander entfernten theokosmischen Räumen mit anderswie ausgerichteten Bindungssystemen besteht die Möglichkeit, dass zwei oder mehrere Personen Gemeinschaftsstrukturen aufbauen, ohne ganz allgemein ihre Zugehörigkeit zum eigenen Raum aufzugeben. Man kann sich dieses dadurch erklären, dass eine bestimmte Bindungsnische so kräfitg aktiviert wird, dass die Hemmungsaktion anderer Bindungsfaktoren überwunden wird. Dieses führt zu einer Umstrukturierung des eigenen Systems, die bis dann dominierenden Bindungen verlieren, wenigstens für eine Zeit, ihre führende Rolle zugunsten einer bis dann wenig bedeutenden oder gar nicht existierenden, die sich impulsartig durchsetzt. Solche Umwälzungen sind nicht ungefährlich, weder für die betreffende Person, noch für den sie tragenden Raum. Es setzt ein Gefühl der Unzufriedenheit ein, das zum Verlassen des in Frage kommenden Raumes und zur Gestaltung eines neuen, selbständigen oder auch eingegliederten Raumes der Gemeinschaft führt. In Extremfällen ist es möglich, dass die sichtbaren Verbindungen mit den ursprünglichen Werten und Bindungen, wenigstens mit ihren wahrnehmbaren Ausdrücken, aufgegeben werden, selbst wenn sie untergründig, zum Teil wenigstens, weiterhin wirksam sind. Die Dauer dieser neuen Struktur hängt von der Stärke der neu entstandenen Bindung, vom Zufriedenheitsgefühl, das sie vermittelt, von ihrer Kraft ab, dem Druck zu widerstehen, der von der ursprünglichen Konfiguration des eigenen Bindungssystems ausgeübt wird.

Diese Situation kennt auch den Extremfall des vollständigen Risses, wenn der eigene theokosmische Raum vollständig und endgültig verlassen wird, wenn man alle bewussten Bindungen zugunsten eines neuen Raumes, 
mit anderen Schwerpunkten, aufgibt. Die direkten Ursachen dieses Ab- und Aufbruchs können unterschiedlich sein, wir können ihnen hier nicht nachgehen. Bemerkenswert ist nur die Tatsache, dass die alten Bindungen im Unterbewussten weiterleben und wirken (die bewussten werden systematisch vermieden und so gut es geht ausgelöscht), aber in einem Gefüge mit anderen Prioritäten. Einen solchen dramatischen Übergang bemerkt man oftmals, nicht immer, bei manchen Konvertiten oder Auswanderern, die in der ehemaligen Beheimatung kaum etwas Positives und nur Negatives erkennen können. ${ }^{13}$

Es gibt noch zahlreiche Möglichkeiten der Gemeinschaft zwischen Personen aus Räumen mit verschiedenen Bindungssytemen, wir werden nur eines davon ins Auge fassen. Im Vordergrund der Situation (also des ursprünglichen Tatbestandes) stehen zwei theokosmische Räume von unterschiedlicher Größe mit weit auseinanderliegenden Wertesystemen, mit ihren entsprechenden, Bindungen. Von dem eventuell zahlenmäßig und gesellschaftlich, oder vielleicht nur wirtschaftlich und politisch mächtigeren Raum gehen kräftige Impulse aus, denen sich die meisten Angehörigen des kleineren Raumes möglicherweise widersetzen.

In einer ersten Variante sind die Mitglieder des kleineren Raumes mit dem eigenen Bindungssystem und den sich daraus ergebenden Gemeinschaften mit anderen, vielleicht übergeordneten Räumen, so unzufrieden und die Anziehungskraft des größeren Raumes ist so groß, dass sich alle Mitglieder des kleineren von Werten und Bindungen so angesprochen fühlen, dass sie die eigenen größtenteils aufgeben und sich die angebotenen aneignen. Es ist praktisch ein totaler Übergang, wie es im Falle einer Massenkonversion geschieht, oder einer neuen kulturellen Strömung, die eine alte, bis dann vorherrschende, ersetzt.

In einer zweiten Variante erkennen die Mitglieder des nicht unbedingt kleineren, aber in irgend einer Hinsicht unterlegenen Raumes, ihr vielleicht kulturelles, wirtschaftliches, meistens politisches Unvermögen, dem Angebot/Angriff des anderen, überlegenen Raumes standzuhalten. So geschieht es in allen Diktaturen. Die Reaktionen darauf sind nicht einheitlich. Die meisten Mitglieder passen sich stillschweigend an und bewahren die Hauptstruktur ihres Bindungssystems, wenigstens eine Zeit lang. Danach folgt unvermeidbar eine steigende „Ansteckung“ mit fremden Bindungen, so dass letztlich, mit einigen Vorbehalten, eine tatsächliche Gemeinschaft mit dem jetzt auch im eigenen Inneren als überlegen anerkannten Raum stattfindet. Leichter sogar mit einigen seiner Mitglieder, aufgrund zwischenmenschli-

\footnotetext{
13 Ich verwende ganz gezielt die Formulierung „erkennen können“, denn die neue Gestalt des Bindungssystems erlaubt ihnen keine neue Perspektive mehr. Ihre neue Verschiedenheit trennt sich von der alten, bekämpft sie sogar blindlings, um sich so durchzusetzen, um nicht zurückzufallen.
} 
cher Beziehungen. Man erkennt in diesem so gezeichneten Bild die Masse der Bevölkerung aus allen Gewaltstaaten, die sich ursprünglich der Situation nur anpasst, um danach Vorzüge zu entdecken, die eigentlich nicht vorhanden sind. Dieses ist nur aufgrund einer subtilen, d. h. nicht erkennbaren Umänderung des eigenen Bindungssystems im Sinne einer Werteverschiebung möglich, deren sie sich nicht bewusst sind.

Eine zweite Reaktion ist total abwehrend, sie bewahrt mit aller Strenge die Struktur und die Ausdrucksformen des Bindungssystems ihres kleineren oder ganz einfach überrumpelten theokosmischen Raumes, und gerät damit in Kollision nicht nur mit den unerwünschten Partnern, sondern oftmals auch mit Mitgliedern ihres eigenen Raumes, die sich inzwischen „angepasst“ haben. In einem demokratischen Land wird dieses Schwimmen gegen den Strom von der Mehrheit ihrer früheren Mitstreiter gegen als ein Epiphänomen betrachtet, das man, vielleicht auch verpönt, schon dulden kann. In einem Gewaltstaat sieht es ganz anders aus: die Widersacher werden so gut es geht zum Schweigen gebracht oder gar physisch beseitigt (Ausweisung, Inhaftierung, Hinrichtung). Von ihren eigenen Werten und den entsprechenden Bindungen getragen, sehen die Machthaber nicht nur keinen Zugang zum Bindungssystem der lästigen Personen, sondern betrachten diese als eine Gefahr für viele der ehemaligen Genossen, weil sie durch ihren Widerstand deren untergetauchte Bindungen wieder ans Licht bringen könnten.

Die dritte Reaktion nähert sich dem goldenen Mittelweg an: Personen, die ganz streng in ihrem Inneren und in einem engen Kreis von Gleichdenkenden ihre Zugehörigkeit zum Bindungssystem ihres ursprünglichen theokosmischen Raumes bekennen, aber nach außen eine „Anpassung“ zeigen, die eigentlich nur peripher existiert. Sie pflegen schon eine Gemeinschaft mit Vertretern des übergeordneten Raumes, aber täuschen nur vor, dessen Werte und Bindungen anzuerkennen. Diese Einstellung hat auch nur unter diktatorischen Bedingungen einen Sinn und stellt eine Abwehrmaßnahme dar, mit deren Hilfe jemand praktisch in zwei sich überquerenden Räumen leben kann, ohne die ihm wichtigen Bindungen aufzugeben und an der Erfüllung neuer Werte teilzunehmen, so weit sie/er sie als solche anerkennt. Die Kryptojuden, die in Spanien nach 1492 ihren Glauben im Geheimen behielten, obwohl sie sich öffentlich zum römischen Katholizimus bekannten, scheinen mir ein typisches Beispiel für diese Überlebensreaktion zu sein.

Allgemeines über Mischehen. Im Zusammenhang der Paradieserzählung hatten wir die Begegnung von Mann und Frau als Gemeinschaft gedeutet, die einen neuen theokosmischen Raum mit zwei Ausgangspunkten begründet, von denen wir jetzt sagen können, dass es zwei Verschiedenheiten sind. Es ist eigentlich ein Topos, denn alle Ehen kommen in gleicher Art und Weise 
zustande: zwei Personen, also zwei Verschiedenheiten, aus unterschiedlichen theokosmischen Räumen, treten in Gemeinschaft und gestalten einen dritten Raum, die Familie. In diesem Sinne sind alle Ehen eigentlich Mischehen. Die gängige Bedeutung des Begriffes ist aber viel enger, sie schließt nur die Situation von Personen aus weit entfernten theokosmischen Räumen ein, deren Gemeinschaft für diese, oder wenigstens für einen Teil ihrer Mitglieder, gerade wegen einer entscheidenden Distanz zwischen den Werte- und Bindungssystemen problematisch oder sogar nicht annehmbar sein kann. Meistens wird der Begriff für Ehen zwischen einer Frau und einem Mann, insbesondere unterschiedlicher religiöser, aber auch nationaler Zugehörigkeit, verwendet. Die Entfernung zwischen den Ursprungsräumen kann aber zahlreiche Ursachen haben. Zum Beispiel, ganz einfach entgegengesetzte Entwicklungen, geographische und kulturelle Entfernung, was bei Begegnung zu Überraschungen und einer gewissen Angst vor dem Unbekannten führen kann. Andere möglichen Ursachen? Die Fehde zwischen zwei Familien, sagen wir Montague und Capulet, die eine Heirat von Romeo und Julia unmöglich macht, um die berühmteste Tragödie dieser Art anzuführen. Klassenunterschiede könnten auch ein gutes Beispiel sein. Der Gegenstand der Spannung zwischen den zwei theokosmischen Räumen ist von Fall zu Fall ein anderer. Die Struktur der Situation ist aber praktisch immer dieselbe, und zwar jene, die wir schon erörtet haben: wenigstens eine hierarchisch hochgestellte und das ganze System prägende Bindung aus dem einen Raum widersetzt sich größtenteils oder sogar total der ihr entsprechenden aus dem anderen.

Nicht in allen Fällen ist die Lage so dramatisch wie in Shakespeares Tragödie, es gibt genügend Mischehen, die ohne Spannung zustande kommen und auch so verlaufen. Der Grund dafür ist ganz einfach - die Verschiedenheiten sind präsent, aber nicht in der Lage, den Impuls zur Gemeinschaft zu hemmen. Ganz im Gegenteil, dieser Impuls schafft eine Bindung, die den möglichen Widerständen der eigenen Bindungssysteme Stand hält und einen emergenten Raum schafft. Andererseits könnten die Ursprungsräume oder die zwei Beteiligten die trennende Schärfe der Bindungen abgeschliffen haben, so dass diese nicht mehr als Gegenkraft eingesetzt werden.

Problematisch wird es aber, wenn die zwei Partner aus Ursprungsräumen stammen, die sich in unterschiedlicher Art und Weise auf einen bestimmten, hierarchisch hochgeschätzten Wert beziehen und von daher auch eine unterschiedliche Bindung dazu entwickeln. Wenn der eine beispielweise als Hindu eine Vielzahl von Göttern anbetet und die/der andere als Muslimin/Muslim diesen Glauben als vom Islam streng verbotene Götzenanbetung betrachtet. Die religiöse Bindung ist in beiden Räumen von entscheidender Bedeutung und die von ihr stammenden Regeln lassen keine eheliche Gemeinschaft zwischen einem Muslimen und einer Hindi 
bzw. einem Hindu und einer Muslimin zu. Ein solches Paar hat aber eine Beziehung aufgebaut und schließt eine Mischehe, was nichts anderes heißt, als das in jedem von ihnen eine Bindung entstanden ist, welche die übliche innere Werte- und Bindungshierarchie ändert. Die Beziehung zu dem möglichen Partner gewinnt die Oberhand, die ihr entgegengesetzten Bindungen werden relativiert oder ganz beseitigt. Wir sahen schon, dass die ehemaligen Bindungen im Unterbewussten weiter leben und einen ununterbrochenen Druck ausüben, sie wollen wieder prägend wirken. Eine mögliche Folge wäre, dass sie kräftig genug sind, die Gemeinschaft und den durch sie aufgebauten theokosmischen Raum der Familie ständig zu belasten oder sogar zu zerstören. Genauso gut ist es aber möglich, dass die Bindungen zwischen den Eheleuten so weit gereift und so fest sind, dass sie gegenseitig ihre Ursprungsbindungen respektieren und helfen, diese zu pflegen.

Nicht weniger kompliziert ist die Situation der Leute aus den theokosmischen Ursprungsräumen. In jedem von ihnen verlangen die nicht legitimen Aufwertungen der trennenden Bindungen die Loslösung von ihrem Mitglied, das ihrer Meinung nach die Regeln des Raumes verletzt hat. Die Kohärenzbindungen des jeweiligen Raumes widersetzen sich aber der Ausweisung und so kann auch hier eine Spannung entstehen, die aufgelöst werden muss. Es wird meistens nach einer Lösung gesucht, die in ganz argen Situationen rein exklusivistisch ist: die Ehe kann nur dann fortdauern, wenn der eine Partner den Glauben des anderen annimmt. Sollte das nicht passieren, wird die Ehe als null und nichtig erklärt und als solche überhaupt nicht anerkannt. Wenn das abtrünnige Glied diese Entscheidung nicht annimmt, so wird es unter Acht und Bann gesetzt, wie das konkret auch aussehen mag. In einer weniger zugespitzten Situation kann die Mischehe so wie sie ist geduldet werden, was dann aber auch bedeutet, dass die in jenem theokosmischen Raum herrschenden trennenden Bindungen keine absolute Oberhand haben, sie werden von jenen, welche die Gemeinschaft fördern, teilweise ausgeglichen.

Im angeführten Beispiel wurde die Gemeinschaft der Ehe, in denen religiöse Bindungen das Wertesystem beherrschen, von Verschiedenheiten gehemmt. Das ist bei Weitem nicht überall der Fall. Bindungen ganz anderer Art, wie z. B. nationale Zugehörigkeit, können dieselbe Rolle spielen. Der Zugriff auf religiöse Bindungen war aber nicht zufällig, denn er ermöglicht uns, auch christliche Mischehen prinzipiell in dieses Argumentationsmodell einzuschließen. Das, was ganz allgemein über Gemeinschaft und Verschiedenheit bzw. über Mischehen gesagt wurde, gilt auch hier. Hinzu kommen noch spezifische Elemente des theokosmischen Raumes „Christentum“, in dem die Überwindung der nicht legitimen Verminderungen und Aufwertungen von Kommunikationswerten in Jesus Christus die zentrale Rolle spielen. 
Wie das geschieht, wie die bestehenden Bindungssysteme durch Christi Leben und Werk erneuert wurden, wie wir daran durch die Gemeinschaft mit Jesus Christus teilhaben, all das kann kein Gegenstand von Vorüberlegungen sein. Diese öffnen aber Horizonte des Verstehens, die nicht nur nach vorne zeigen, sondern alle nachparadiesischen Räume einbeziehen. Christi Wirken erstreckt sich in geheimnisvoller Art und Weise auf jeden von ihnen aus, auf die bestehenden oder nicht bestehenden Gemeinschaften ihrer Verschiedenheiten. Wie das erfolgt und was für eine Bewandtnis es für die Christen von heute und von morgen, für ihre Ehen und Mischehen hat, das möchten wir zum Gegenstand einer anderen Untersuchung machen. 